\title{
The Design and Research of the Smart Path Tracing Car Based on the Im- age Recognition
}

\author{
Chen Meng-yuan*
}

Anhui Polytechnic University, Key Laboratory of Anhui Electric Drive and Control, 241000, Wuhu, Anhui, China

\begin{abstract}
To realize the smart car's automatic path tracing along the different types of path, a smart path tracing car based on car model is developed. In the design of the smart path tracing car, image processing affects the smart car's performance directly. This article adopts direct acquisition, direct edge detection algorithm and the improved edge detection algorithm to analyze and compare the road message acquired by COMS camera. Plenty of experiment data show that, under different light intensity, the edge detection algorithm can acquire image with better accuracy and efficiency, solving the contradiction between the system's rapidity and stability, and realizing the smart car's running along the guide curve stably and rapidly.
\end{abstract}

Keywords: Smart car, Camera, Edge detection, Path identification.

\section{INTRODUCTION}

Path tracing is one of key technologies for intelligence robots to realize autonomous motion, and visual navigation is one of the methods which possess good stability among several existing navigation methods. In this article, a standard car model was studied and the road image was dynamically gained through the vehicle-borne CMOS camera. The smart car can run along the black, zonal and car-centered laid guide curve automatically under swerve and speed control on a white KT board with specific geometric constraints, friction coefficient and optical properties, which help spend minimum time fulfilling the tracing task on the premise of keeping the system stable.

In the aspect of path recognition, uniform illumination is usually considered while the influences on the image acquisition from the illumination intensity is usually neglected, which causes the loss of image information and the decrease on the accuracy of smart car's path judgment [1-8]. This article applies the improved edge detection algorithm in the image processing and adopts dynamic threshold to improve the dipartite degree of the image and the accuracy of image feature extraction caused by uneven distribution of light intensity during the course of the smart car's moving ahead.

\section{THE HARDWARE DESIGN OF TRACING SMART CAR}

The smart tracing car takes Freescale's MC9S12XS128 microprocessor as its controlling core. Moreover, the system includes power management module, image acquisition module, motor driving module, speed detection module and

*Address correspondence to this author at the Anhui Polytechnic University, Key Laboratory of Anhui Electric Drive and Control, 241000, Wuhu, Anhui, China; E-mail: mychen@ahpu.edu.cn actuator steering module, etc. The construction of the system is shown in the Fig. (1).

The Freescale MC9S12XS128, a microprocessor which sets the system's working frequency that can reach maximum 96 MHZ by phase-locked loop (PLL), is taken as smart path tracing car's central control chip. The car adopts 64 MHZ operating frequency to satisfy the requirement of CMOS camera's information acquisition. Meanwhile, PWM module is used to control actuator's steering angle and motor's speed while Enhanced Capture Timer (ECT) is responsible for encoder pulse counts. Besides, asynchronous serial port (SCI) sent the key parameters gathered in the process of running to $\mathrm{PC}$ by the ZigBee wireless communication dynamic technology, which makes real-time analysis convenient and feasible. According to the plentiful image parameters, the microprocessor's high-speed synchronous serial interface (SPI) is used to send them to the SD card timely to get image information in the process of the whole running.

The image acquisition module adopts digital camera OV6620 based on CMOS sensor. Moreover, collected images are based on PAL with $25 \mathrm{fps}$, two games per frame, $356 \times 292$ pixel per picture. Camera parameters such as the biggest grayscale, contrast and exposure rate can be modified through the internal register. Since the smart car can just identify the black guide line on the way, what we merely need is image grayscale information. The gray value is sent to MC9S12XS128's common data port through OV6620 camera's pin Y0 Y7 in parallel. The clock control signal field interrupt signal (VSYN) and line interrupt signal (HREF) of the OV6620 camera that is linked to the PT1 and PT0 pin in enhanced capture timer (ECT) module of the microprocessor respectively.

Due to the complex path, the smart car has to constantly adjust its direction to adapt to the changeable runway. The actuator steering module serves as actuating mechanism to control direction of the smart car. The microprocessor ana- 


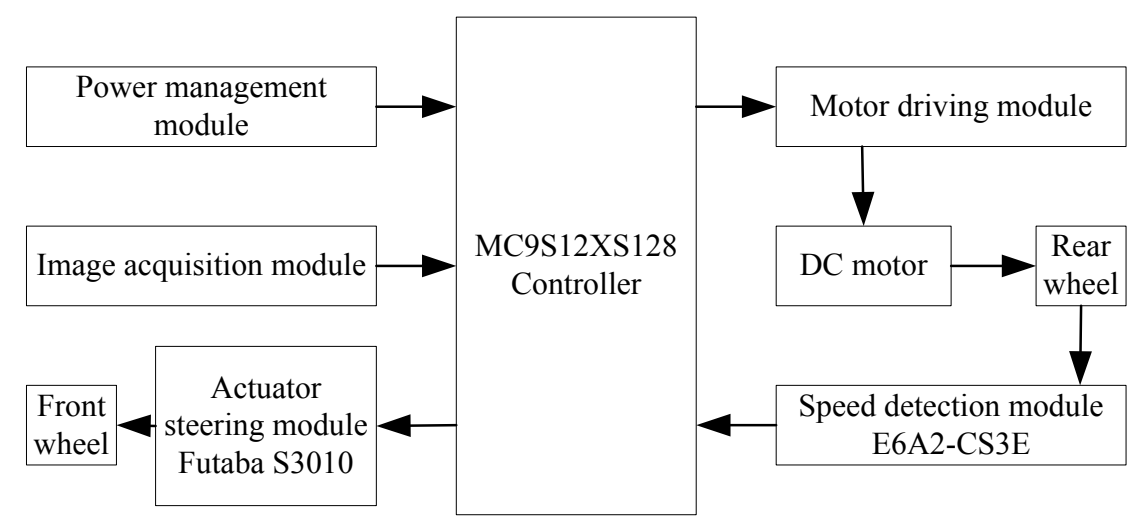

Fig. (1). The general structure diagram of system.

lyzes and deals with the traffic information collected from the camera, controlling the steering motor to meet corresponding turning requirements based on different acquisition signal, ensuring the intelligent car can move ahead along the track accurately.

To meet the power requirements in the rapid movement of the smart car, the motor choose BTS7960B chip, a kind of high current half bridge drive chip with 43A highest current that can achieve, to realize thermal shutdown function and low consumption due to its small internal resistance whose typical value is $16 \mathrm{M} \Omega$. In this paper, the BTS7960B chip converts constant battery voltage to a PWM pulse voltage sequence with constant frequency and variable width. The motor's rotation speed is changed through adjusting the value of average voltage. In order to improve the control precision, the internal two way 8-bit PWM registers of the MC9S12XS128 are cascaded as one way 16-bit register, which increased the control accuracy 256 times than before. After the concatenation, the output pulse of PWM3 and PWM5 pin connects BTS7960B's input IN through buffer driver 74HC244, and BTS7960B's output terminal OUT1 and OUT2 is connected to motor's one edge after being paralleled, achieving the purpose of controlling motor's rotating and reversing.

The Omron's E6A2 - CS3E photoelectric encoder is used to test the current speed of the smart car, and the feedback is preached to the microprocessor. Thus the closed-loop control on speed is realized through various intelligent algorithms.

\section{THE IMAGE PROCESSING OF THE SMART TRACING CAR}

The image processing distinguishes the position of the black guide line through the digital signal (that is gray value) from camera. The common algorithms which can extract black line's information include binarization and direct edge detection algorithm [2-13]. However, camera pixel's gray value is greatly influenced by light, that is to say, when light gets stronger, the gray value of black and white pixels will get higher. On the contrary, the gray value will get smaller. In the case of uneven illumination, the two thresholds value is difficult to determine when the binary algorithm is used to process the image. Furthermore, the image can hardly find the black guide line when the light changed slightly. Although the direct edge detection algorithm is suitable for the occasion in which the light changes weakly, when the light changes sharply, the gray value of the black and white pixel will not be linearly changed, while the black line of the image cannot be distinguished effectively. This design ameliorates the edge detection algorithm, adopting the dynamic threshold value instead of the original static threshold. The formula to calculate the dynamic threshold value is shown in formula (1) [14-18].

$$
T(i)=\lambda\left(\frac{\sum_{m=0}^{k} X(m)}{k+1}-\frac{\sum_{n=0}^{l} X(n)}{l+1}\right)
$$

In the formula, $\mathrm{T}(\mathrm{i})$ is a dynamic threshold which need to be calculated in the $i$ line; $X(m)$ is the gray value of white pixel in the $i$ line; $X(n)$ is the gray value of black pixel in the $i$ line; $\lambda$ is threshold coefficient generally ranging from 0.7 to 1.0. First, It is natural to subtract the two geometric means, which are calculated from the white pixel's gray value and the black pixel's gray value in previous row, to serve as new threshold of the next row. Due to the different light intensity in different occasion, the thresholds are different from each other, the disparity in gray value between the two adjacent lines is small, so the dynamic threshold value extracts the image information from black line more effectively and accurately [19-22].

In order to compare the processing effect on image based on the two types of edge detection algorithm practically, the VC software is used to develop the upper computer and restore the collected image data.

The runway of the smart car is generally composed of straightaway, square corners and $\mathrm{S}$ type curves which are shown in Fig. (2) [22-26].

Fig. (3-5a) is a set of images gathered from the experiment. In the image, the shadow part in the middle is the black guide line and the bright spot is interference spot. Fig. (3-5b) is the processed image based on direct edge detection 


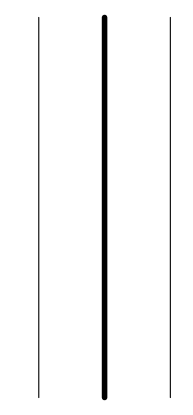

(a) Straightaway

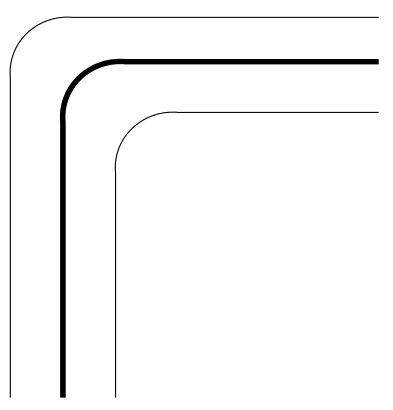

(b) square corners

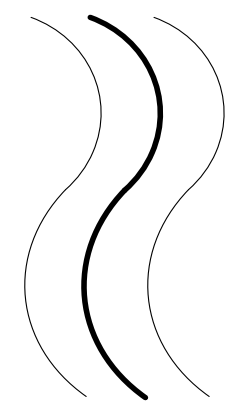

(c) S type curves

Fig. (2). Path types.

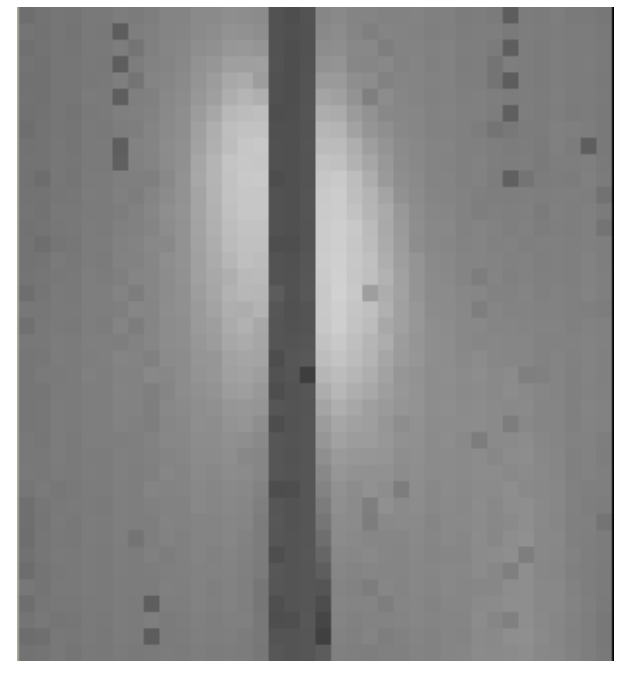

(a) The collected image

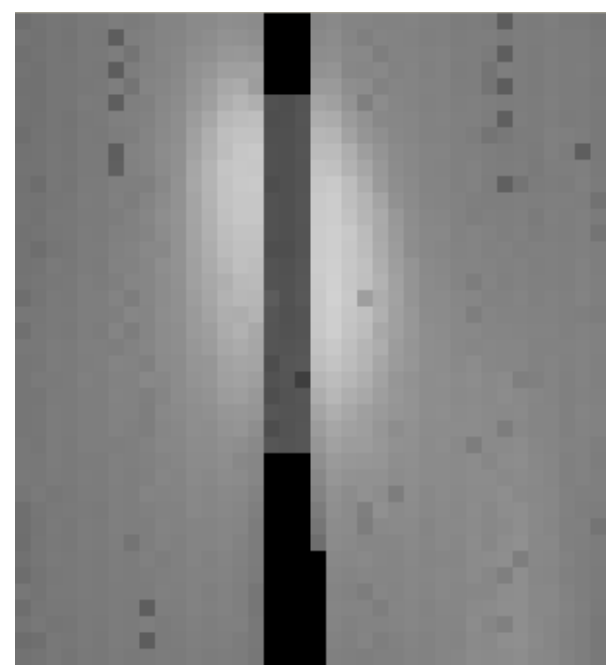

(b) The image after processing based on direct edge detection algorithm 


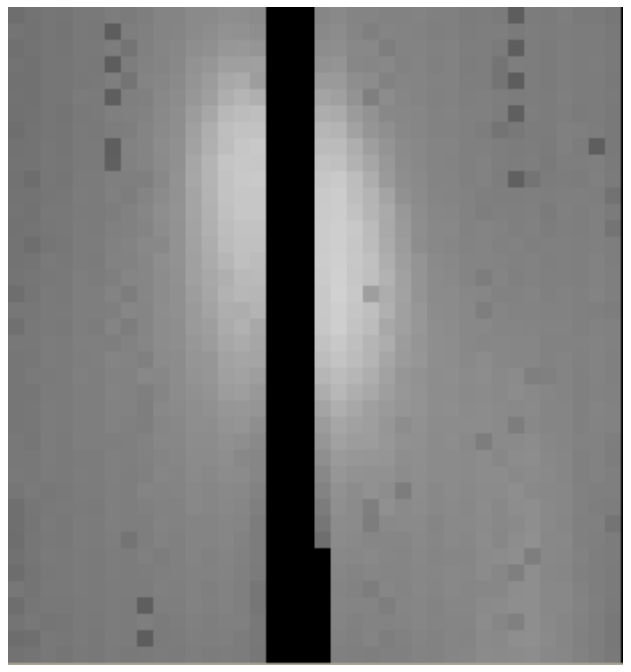

(c) The image after processing based on the improved edge detection algorithm

Fig. (3). The comparison diagram of straightaway before and after the image processing.

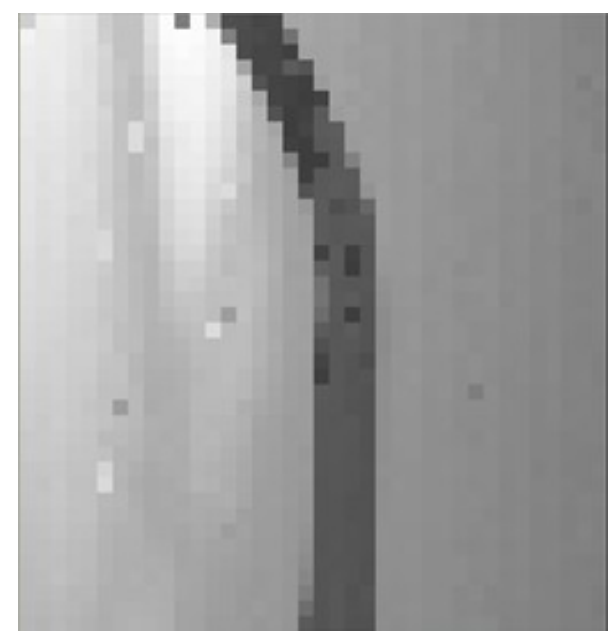

(a) The collected image

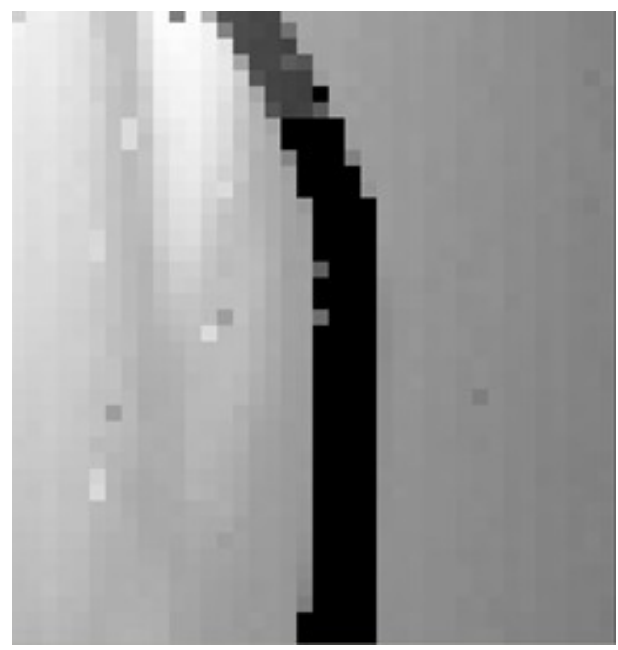

(b) The image after processing based on direct edge detection algorithm

Fig. (4). Contd... 


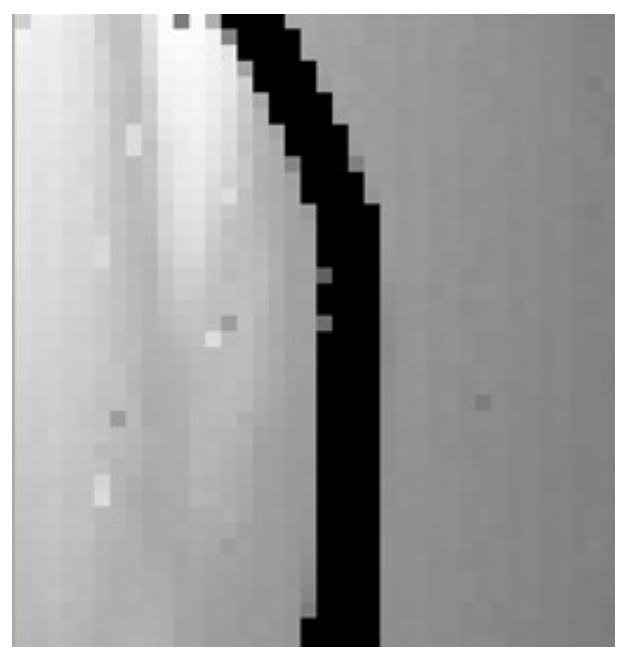

(c) The image after processing based on the improved edge detection algorithm

Fig. (4). The comparison diagram of the square corners before and after the image processing.

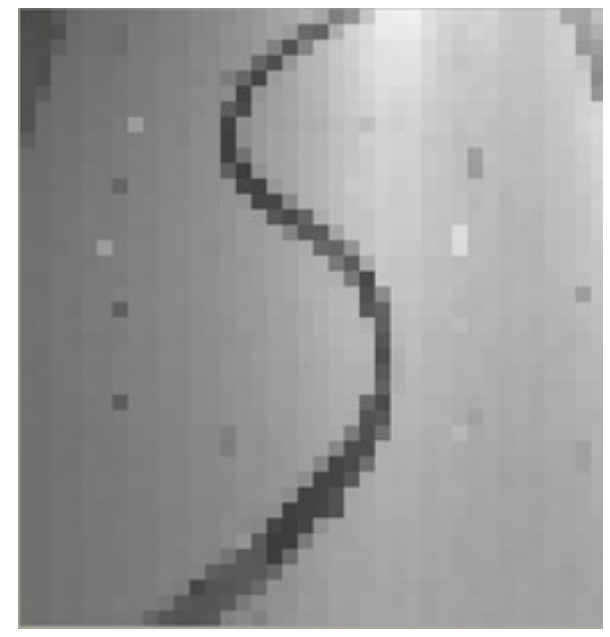

(a) The collected image

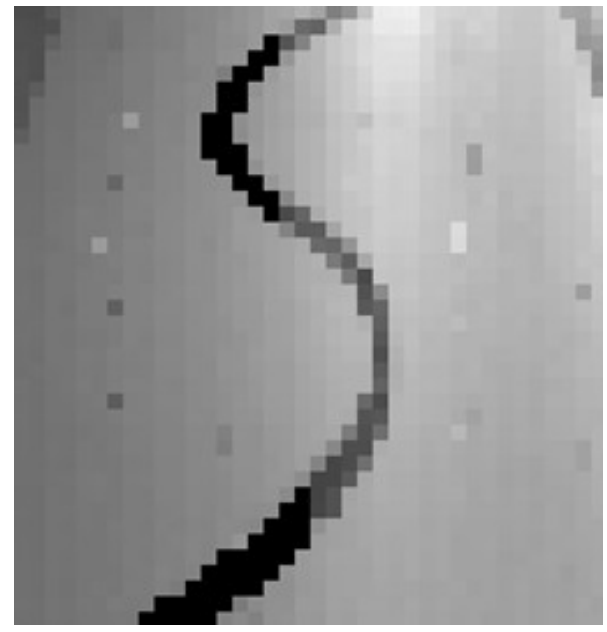

(b) The image after processing based on direct edge detection algorithm

Fig. (5). Contd... 


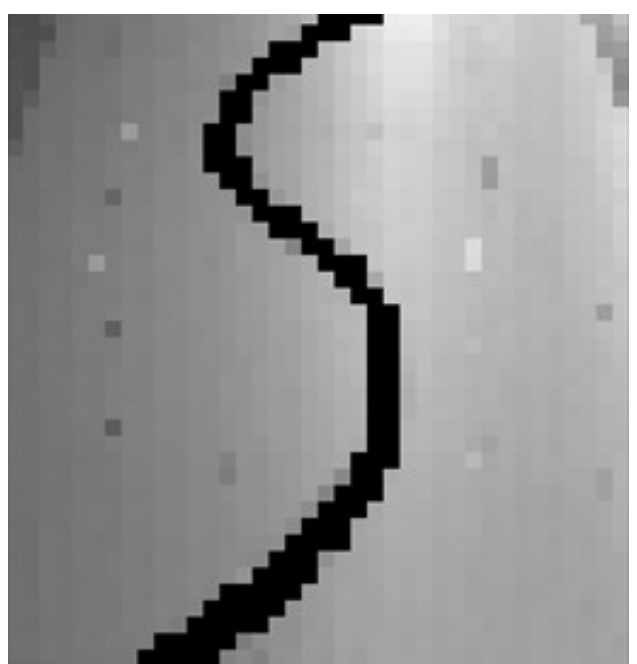

(c) The image after processing based on the improved edge detection algorithm

Fig. (5). The comparison diagram of $\mathrm{S}$ type curves before and after the image processing.

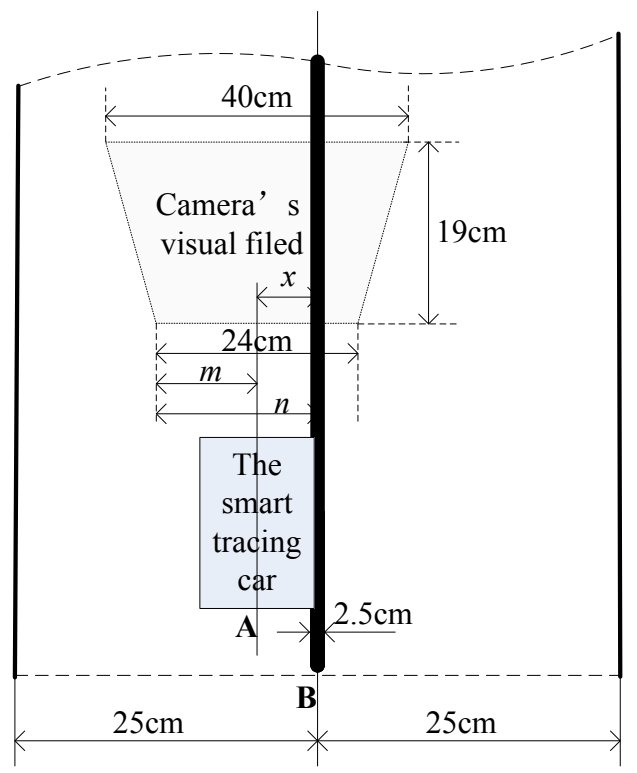

Fig. (6). The schematic diagram of measurement on experimental parameters.

algorithm and Fig. (3-5c) is the processed image based on the improved edge detection algorithm. By comparison, it is shown that direct edge detection algorithm cannot distinguish the black line accurately when meeting severe fluctuating illumination; nevertheless, the improved edge detection algorithm can adapt to the change of light and distinguish the black line on the path.

\section{THE EXPERIMENT SCHEME AND ANALYSIS}

The experiment parameter of the smart car, such as the speed and the deviation between the car's geometric center and the road's center, must be measured in the course of driving. Therefore, data of every field from gathered images should be stored in the SD card, then these image data will be restored one by one through the upper computer and the experiment parameters can be measured. The Fig. (6) shows the measurement of experimental parameters. As Fig. (6) shows, the straight line containing point $\mathrm{A}$ is the geometric center of the smart car, the straight line containing point $\mathrm{B}$ is the geometric center of the road. The width of the path is $50 \mathrm{~cm}$ and the width of the black guide line is $2.5 \mathrm{~cm}$. The square in the schematic diagram represents the smart car and the trapeziform with dotted line represents the visible range of camera which also means the image saved to SD card [27, 28]. The vision width between the image and the smart car ranges from $24 \mathrm{~cm}$ to $40 \mathrm{~cm}$. The experiment chooses one line from the image to measure the distance ' $\mathrm{m}$ ' from the field's leftmost end to the center of the field (namely the geometric center of the smart car) and the distance ' $n$ ' from the field's right end to the center of the black line (that is the center of the road), then actual deviation of ' $x$ ' $(x=|n-m|)$ can be gained after proportional reduction [29]. 
Table 1. The tracking error of the image acquisition in uniform illumination.

\begin{tabular}{|c|c|c|c|c|c|c|}
\hline & \multicolumn{3}{|c|}{ Mean (cm) } & \multicolumn{3}{c|}{ Variance $\left(\mathrm{cm}^{2}\right)$} \\
\hline & $\mathbf{0 . 8 ( m / s )}$ & $\mathbf{1 . 2 ( m / s )}$ & $\mathbf{1 . 6}(\mathbf{m} / \mathbf{s})$ & $\mathbf{0 . 8}(\mathbf{m} / \mathbf{s})$ & $\mathbf{1 . 2}(\mathbf{m} / \mathbf{s})$ & $\mathbf{1 . 6 ( m / s )}$ \\
\hline \hline Straightway & 0.83 & 1.50 & 2.16 & 1.8333 & 3.1667 & 6.5000 \\
\hline Square corners & 3.00 & 3.50 & 4.33 & 11.6667 & 20.1667 & 26.0000 \\
\hline S type curves & 3.50 & 4.17 & 5.50 & 18.5000 & 25.1667 & 43.1667 \\
\hline
\end{tabular}

Table 2. The tracking error of the image acquisition after the treatment of improved edge detection algorithm in uniform illumination.

\begin{tabular}{|c|c|c|c|c|c|c|}
\hline & \multicolumn{3}{|c|}{ Mean (cm) } & \multicolumn{3}{|c|}{ Variance $\left(\mathrm{cm}^{2}\right)$} \\
\hline & $0.8(\mathrm{~m} / \mathrm{s})$ & $1.2(\mathrm{~m} / \mathrm{s})$ & $1.6(\mathrm{~m} / \mathrm{s})$ & $0.8(\mathrm{~m} / \mathrm{s})$ & $1.2(\mathrm{~m} / \mathrm{s})$ & $1.6(\mathrm{~m} / \mathrm{s})$ \\
\hline Square corners & 2.33 & 2.67 & 3.50 & 7.6667 & 12.6667 & 16.8333 \\
\hline
\end{tabular}

The results of the experiment show that the maximum speed of the smart car can reach $3.5 \mathrm{~m} / \mathrm{s}$. In order to obtain more stable parameters, the deviation of three path types containing straightway, square corners and s type curves are recorded respectively when the smart car runs at $0.8 \mathrm{~m} / \mathrm{s}$, $1.2 \mathrm{~m} / \mathrm{s}$ and $1.6 \mathrm{~m} / \mathrm{s}$. The curvature radius of the curve is $50 \mathrm{~cm}$. The mean and variance can be obtained through calculating the deviation. The mean reacts to the deviating distance from the smart car to the center of the black line while the variance reacts to the stability of the smart car.

The smart tracing car runs on the basis of the collected image processed in the case of direct acquisition and edge detection algorithm respectively under uniform illumination; experimental data that can be obtained is shown in Table 1 and Table 2.

As Table 1 shows, when the smart car runs at a speed of $0.8 \mathrm{~m} / \mathrm{s}$, the car operates stably on every type of path, meaning the car rarely deviates from the center of the road. Furthermore, when the smart car runs at a speed of $1.6 \mathrm{~m} / \mathrm{s}$, the car also can keep stable operating in straightway with little deviation. However, the smart car will seriously deviate from the center of the road with large tracking error when rounding the square corners and $\mathrm{S}$ type curves at $1.6 \mathrm{~m} / \mathrm{s}$. By comparing the deviation means, a conclusion can be drawn that the smart car has larger deviation on the square corners and S type curves. In order to decrease the deviation originating from the two types of path, predictive control is adopted to make the smart car swerve in advance. By comparing the variance during running, it is known that the large variance reacts in that the dramatic change of the smart car's status has produced certain oscillation.

Taking the case of running on the square corners as an example, the mean square deviation of the path's deviation is $11.6667,20.1667$ and 26.0000 when the car runs at
$0.8 \mathrm{~m} / \mathrm{s}, 1.2 \mathrm{~m} / \mathrm{s}$ and $1.6 \mathrm{~m} / \mathrm{s}$ respectively based on direct acquisition. However, the mean square deviation is $7.6667,12.6667,16.8333$ on the basis of the improved edge detection algorithm, which means the stability of the smart car is increased by $34.2 \%, 37.2 \%, 35.3 \%$ respectively. The results indicate that the smart car based on the improved edge detection algorithm has better stability and smaller mean square deviation if running on the same type of path and at the same speed.

The smart tracing car runs following the image processed from the direct edge detection algorithm and the improved edge detection algorithm respectively under different light intensity. The data obtained in the experiment is shown in Table 3 and Table 4.

By contrasting Table $\mathbf{3}$ and Table $\mathbf{4}$, it is known that data in Table 4 is superior to Table $\mathbf{3}$ when the car runs in the same types of road and at the same speed. It is suggested that, due to the influence of exotic light, the smart tracing car based on direct edge detection algorithm can run along the path but deviate far from the center of the black line with poor stability. Nevertheless, based on the improved edge detection algorithm, the smart car can run closer to center of the black line with better stability, and the error level is close to that in Table 2. By using the improved edge detection algorithm, the large error caused by different light intensity can be decreased and the stability of the smart tracing car is improved.

\section{SUMMARY}

The experimental results show that it is prospective to take the MC9S12XS128 as control core and adopt CMOS camera as sensor to recognize the path, which can realize fuzzy control algorithm through making full use of fuzzy 
Table 3. The tracking error of the smart tracing car after processing based on direct edge detection algorithm in uniform illumination.

\begin{tabular}{|c|c|c|c|c|c|c|}
\hline & \multicolumn{3}{|c|}{ Mean (cm) } & \multicolumn{3}{|c|}{ Variance $\left(\mathrm{cm}^{2}\right)$} \\
\hline & $0.8(\mathrm{~m} / \mathrm{s})$ & $1.2(\mathrm{~m} / \mathrm{s})$ & $1.6(\mathrm{~m} / \mathrm{s})$ & $0.8(\mathrm{~m} / \mathrm{s})$ & $1.2(\mathrm{~m} / \mathrm{s})$ & $1.6(\mathrm{~m} / \mathrm{s})$ \\
\hline Straightway & 1.33 & 1.83 & 2.66 & 4.0000 & 4.5000 & 9.0000 \\
\hline Square corners & 3.33 & 3.83 & 4.67 & 13.3333 & 24.8333 & 30.6667 \\
\hline S type curves & 3.83 & 5.00 & 6.00 & 20.5000 & 33.5000 & 52.3333 \\
\hline
\end{tabular}

Table 4. The tracking error of the smart tracing car after the treatment based on the improved edge detection algorithm in case of different light intensity.

\begin{tabular}{|c|c|c|c|c|c|c|}
\hline & \multicolumn{3}{|c|}{ Mean (cm) } & & \multicolumn{2}{c|}{ Variance (cm $\left.{ }^{2}\right)$} \\
\hline & $\mathbf{0 . 8 ( m / s )}$ & $\mathbf{1 . 2 ( m / s )}$ & $\mathbf{1 . 6 ( m / s )}$ & $\mathbf{0 . 8 ( m / s )}$ & $\mathbf{1 . 2}(\mathbf{m} / \mathbf{s})$ & $\mathbf{1 . 6 ( m / s )}$ \\
\hline \hline Straightway & 0.83 & 1.33 & 2.17 & 1.8333 & 2.3333 & 5.3333 \\
\hline Square corners & 2.83 & 3.00 & 4.00 & 9.5000 & 14.6667 & 21.6667 \\
\hline S type curves & 3.33 & 4.00 & 4.83 & 16.0000 & 24.6667 & 33.5000 \\
\hline
\end{tabular}

adaptive PI control. On the other hand, adopting different control parameter according to different traffic types can make the smart path tracing car possess a good prejudgment effect and run smoothly along the guide line.

In the aspect of image processing, during the smart car's running, by comparing the error from image processed respectively under the direct edge detection algorithm and the improved edge detection algorithm in the uniform illumination and different light intensity situations, it is found that adopting the improved edge detection algorithm can well eliminate the negative influence on the image caused by different light intensity and improve the anti-interference ability of the smart car. Moreover, it helps increase the average speed of the smart car from $1.4 \mathrm{~m} / \mathrm{s}$ up to $2.1 \mathrm{~m} / \mathrm{s}$.

\section{CONFLICT OF INTEREST}

The author confirms that this article content has no conflict of interest.

\section{ACKNOWLEDGEMENTS}

Declared none.

\section{REFERENCES}

[1] H. Xu, and J. Tang, "Light modulation line-tracking system and it's application in a autorunning robot," Journal of Tsinghua University, vol.1, no. 42, pp. 115-117, 2002.

[2] Y. Zhang, E. Shi, C. Wu, and Z. Qin, "On the navigation system based on CCD for smart car," Northeastern University, vol. 2, no. 30, pp. 162-165, 2009.

[3] J. Zheng, L. Huang, and P. Ge, "Autonomous tracing in intelligent vehicle based on CCD," Journal of Donghua University, vol. 6, no. 34, pp. 728-730, 2008.
[4] S. Sun, L. Lang, and H. Wang, "The design and implementation of tracing smart car based on path identification," Journal of Anhui Polytechnic University, vol. 1, no. 27, pp. 60-63, 2012.

[5] H. Lan, X. Li, and Y. Ding, "Research on design of fuzzy controller of smart car steering system," Transducer and Microsystem Technologies, vol. 5, no. 29, pp. 34-36, 2010.

[6] T. Qiu, Z. Xu, and H. Jiang, "Smart car road status recognition based on fuzzy control," Computer Engineering and Applications, vol. 14, no. 45, pp. 213-216, 2009.

[7] L. You, and Q. Hu, "Fuzzy control in smart car system with dual steering gears," Computer Engineering and Applications, vol. 32, no. 45, pp. 209-211, 2009.

[8] M. Bertozzi, and A. Broggi, "Gold: a parallel real-time stereo vision system for generic obstacle and lane detection," Image Processing, vol. 1, no. 7, pp. 62-81, 1988.

[9] E. Dickmanns, and B. Mysliwetz, "Recursive 3D road and relative ego-state recognition,' IEEE Transaction on Pattern Analysis and Machine Intelligence, vol. 2, no. 14, pp. 199-213, 1992.

[10] T. Hong, M. Shneier, and R. Chellappa, "A learning approach towards detection and tracking of lane markings," IEEE Transaction on Intelligent Transportation Systems, vol. 13, no. 3, 2012, pp. 1088-1098.

[11] X. Shi, B. Kong, and F. Zheng, "A new lane detection method based on feature pattern," Image and Signal Processing, $2^{\text {nd }}$ International Congress, 2009, pp. 1-5.

[12] T. Y. Sun, and W. C. Huang, "Embedded vehicle lane-marking tracking system," Consumer Electronics, IEEE $13^{\text {th }}$ International Symposium, 2009, pp. 627-631.

[13] L. W. Tsai, J. W. Hsieh, C. H. Chuang, and K. C. Fan, "Lane detection using directional random walks," Intelligent Vehicles Symposium, 2008, pp. 303-306.

[14] H. Shen, S. Li, F. Bo, X. Miao, F. Li, and W. Lu, "Intelligent vehicles oriented lane detection approach under bad road scene," Computer and Information Technology, $9^{\text {th }}$ IEEE International Conference, 2009, pp.177-182.

[15] J. Gonzalez and U. Ozguner, "Lane detection using histogrambased segmentation and decision trees," Intelligent Transportation Systems, 2000, pp. 346-351.

[16] W. Liu, H. Zhang, B. Duan, H. Yuan, and H. Zhao, "Vision-based realtime lane marking detection and tracking," In: $11^{\text {th }}$ International IEEE Conference on Intelligent Transportation Systems, 2008, pp. 49-54. 
[17] M. Bertozzi and A. Broggi, "Gold: a parallel real-time stereo vision system for generic obstacle and lane detection," Image Processing, vol. 7, no. 1, pp. 62-81, 1988 .

[18] Q. B. Truong, B. R. Lee, N. G. Heo, and J. Y. Young, "Lane boundaries detection algorithm using vector lane concept," In: $10^{\text {th }}$ International Conference on Control Automation Robotics \& Vision, 2008, pp. 2319-2325.

[19] M. Meuter, S. Muller-Schneiders, A. Mika, S. Hold, C. Nunn, and A. Kummert, "A novel approach to lane detection and tracking," In: $12^{\text {th }}$ International IEEE Conference on Intelligent Transportation Systems, 2009, pp. 1-6.

[20] G.F. McLean, and D. Kotturi, "Vanishing point detection by line clustering," IEEE Transactions on Pattern Analysis and Machine Intelligence, vol. 17, no. 11, pp. 1090-1095, 1995.

[21] C. V. Stewart, "Robust parameter estimation in computer vision," Silam Review, vol. 41, no. 3, pp. 513-537, 1999.

[22] H. S. Sawhney, S. Ayer, and M. Gorkani, "Model-based 2D and 3D dominant motion estimation for mosaicking and video representation," In: The $5^{\text {th }}$ International Conference on Computer Vision, 1995, pp. 583-590.

[23] E. Dickmanns and B. Mysliwetz, "Recursive 3D road and relative ego-state recognition," IEEE Transaction on Pattern Analysis and Machine Intelligence, vol. 14, no. 2, pp. 199-213, 1992.
[24] N. Apostoloff and A. Zelinsky, "Robust vision based lane tracking using multiple cues and particle filtering," IEEE Intelligent Vehicles Symposium, 2003, pp. 558-563.

[25] Z. Kim, "Robust lane detection and tracking in challenging scenarios," IEEE Transaction on Intelligent Transportation Systems, vol. 9, no. 1, pp. 16-26, 2008.

[26] T. Hong, M. Shneier, and R. Chellappa, "A learning approach towards detection and tracking of lane markings," IEEE Transaction on Intelligent Transportation Systems, vol. 13, no. 3, pp. 10881098, 2012.

[27] C.Y. Chang, and C.H. Lin, "An efficient method for lane-mark extraction in complex conditions," In: $9^{\text {th }}$ International Conference on Ubiquitous Intelligence and Computing and $9^{\text {th }}$ International Conference on Autonomic and Trusted Computing, 2012, pp. 330336.

[28] K. Smith, D. Gatica-Perez, J. Odobez, and S. Ba, "Evaluating multi-object tracking," In: IEEE Computer Society Conference on Computer Vision and Pattern Recognition, vol. 19, no. 5, pp. 10631069, 2005.

[29] Y. Wang, D. Shen, and E. K. Teoh, "Lane detection using catmullrom spline," In: IEEE International Conference on Intelligent Vehicles, 1998, pp. 51-57.

This is an open access article licensed under the terms of the Creative Commons Attribution Non-Commercial License (http://creativecommons.org/licenses/bync/4.0/) which permits unrestricted, non-commercial use, distribution and reproduction in any medium, provided the work is properly cited. 